\title{
Lassa fever: History, causes, effects, and reduction strategies
}

\author{
Promise Tewogbola1 ${ }^{(1)}$ and Norah Aung ${ }^{2}$ \\ 1. School of Psychological and Behavioral Sciences, Southern Illinois University, Carbondale, 62901, USA; 2. Department \\ of Health Sciences and Social Work, Western Illinois University, Macomb, 61455, USA. \\ Corresponding author: Promise Tewogbola, e-mail: promise.tewogbola@siu.edu \\ Co-author: NA: he-aung@wiu.edu \\ Received: 24-12-2019, Accepted: 17-06-2020, Published online: 25-08-2020
}

doi: www.doi.org/10.14202/IJOH.2020.95-98 How to cite this article: Tewogbola P, Aung N (2020) Lassa fever: History, causes, effects, and reduction strategies, Int. J. One Health, 6(2): 95-98.

\begin{abstract}
Lassa fever is a disease that is not well-known worldwide, particularly due to the inability of the multimammate rat, the primary vector of the Lassa virus, to breed in temperate regions. The aim of this review is to provide an overview of the disease and its modus operandi while also providing information about trends in the past decade, as well as proven strategies that have been used to manage its spread.
\end{abstract}

Keywords: Arenavirus, epidemic, epidemiology, Lassa fever, Lassa virus, virus.

\section{Introduction}

Lassa fever, also known as Lassa hemorrhagic fever, is an acute and often fatal viral hemorrhagic fever that is caused by the Lassa virus, which is an enveloped RNA virus from the Arenavirus family of viruses [1,2]. The virus is transmitted to humans through contact with foods and household items that have been contaminated by a zoonotic reservoir, which is usually an infected multimammate rat $[1,3]$. There is also evidence that humans can be infected by being exposed to aerosols from vectors' excreta [4]. According to the US Federal Select Agent Program, the Lassa virus has been classified as a select agent, requiring biosafety level-4 conditions before it can be studied in a laboratory [2]. Lassa fever is endemic in many parts of Nigeria, as well as countries found in the Mano River Union, particularly Sierra Leone, Guinea, and Liberia [2]. Cases have also been reported in Ghana, Mali, and Benin [5]. Lassa fever is notorious for being nosocomial in nature [1], which implies that it can be a hospital-acquired disease. According to the United States Centers for Disease Control and Prevention (CDC), approximately 100,000-300,000 individuals are infected with Lassa fever every year, with about 5000 deaths occurring within the same period [6]. However, since surveillance efforts are not uniform within the regions where the Lassa fever is endemic [1], the estimates of infected individuals are crude and possibly underreported to a large extent. Furthermore, surveillance efforts have been biased toward Lassa fever endemic countries, and as a result, there is lower awareness of Lassa fever outside the

Copyright: Tewogbola and Aung. This article is an open access article distributed under the terms of the Creative Commons Attribution 4.0 International License (http://creativecommons.org/ licenses/ by/4.0/), which permits unrestricted use, distribution, and reproduction in any medium, provided you give appropriate credit to the original author(s) and the source, provide a link to the Creative Commons license, and indicate if changes were made. The Creative Commons Public Domain Dedication waiver (http:// creativecommons.org/ publicdomain/zero/1.0/) applies to the data made available in this article, unless otherwise stated. endemic regions, potentially allowing past human outbreaks to go undetected [7].

The aim of this review is to provide an overview of the disease and its modus operandi while also providing information about trends in the past decade, as well as proven strategies that have been used to manage its spread.

\section{History}

Even though there have been descriptions of symptoms similar to Lassa fever as early as the 1950s in Sierra Leone [8], the virus causing the illness was not identified until 1969 in Lassa, a small town in Borno State, Nigeria, when a missionary nurse died a week after being presumably infected by an obstetrical patient resident in the region. Two other missionary nurses who had managed the first victim also got infected, with one of them eventually succumbing to the viral infection [9]. All through the 1970s and the 1980s, there were numerous in-depth studies of the ecological and clinical significances of the Lassa fever virus carried out in West Africa, particularly in Sierra Leone and Nigeria [10].

Mali reported its first case of Lassa fever in 2009 when it was diagnosed after the death of a British man with a 10-day fever history, who was presumed to be working in Soromba, a village in South Mali $[1,10]$. On the other hand, the first cases of Lassa fever in Ghana were not reported until 2011 when a man from the Ashanti region was presumed to have been infected after consuming contaminated rodents which he had hunted [10]. There have been reports of a few imported cases in Germany, the Netherlands, Sweden, the US, and the UK [11].

\section{Virology}

The Lassa virus is a single-stranded RNA virus belonging to the Arenavirus family, which are known to cause silent and persistent infections in rodents [12]. When compared to other members of 
the Arenavirus family, Lassa virus stands out due to its non-conventional and uncharacteristic serology, genetics, and pathology. The Lassa virus is also genetically diverse, and this makes it difficult to develop a vaccine for the virus. The Lassa virus has a spherical shape with a diameter that ranges from 70 to $150 \mathrm{~nm}$ and a smooth surface envelope with T-shaped spikes and inbuilt glycoproteins. Within the surface envelope of the Lassa virus, there is a nucleocapsid, which contains the genome of the virus and ranges between 400 and $1300 \mathrm{~nm}$ in length [9]. The Lassa virus has at least four lineages, with three found in Nigeria and the fourth one reported in Guinea, Liberia, and Sierra Leone [13]. There has also been a proposed fifth Lassa virus lineage that has been reported in Mali and Cote d'Ivoire [1].

\section{Reservoir Species}

The primary hosts for the Lassa virus is the multimammate rat (Mastomys natalensis) which is one of the most prevalent rodent species that is found in Sub-Saharan Africa. Despite the fact that they are capable of reproducing throughout the year, the multimammate rats produce fewer offspring in the rainy season, which may be due to the flooding that is more probable at this time of the year [14]. Even though the multimammate rat serves as the animal reservoir for the Lassa virus, it does not become sick as a result of the viral infection [5]. Reports have shown that Lassa virus transmission in multimammate rats occurs both horizontally and vertically, with the horizontal transmission occurring more often than vertical transmission [2]. There is also emerging evidence that both the African wood mouse Hylomyscus pamfi and the Guinea multimammate mouse Mastomys erythroleucus can serve as reservoir host species to the Lassa virus [15]. Multimammate rats are more rampant in rural regions, and in lesser numbers in forested and urban areas, their presence is a good predictor of the likelihood of humans being infected with the Lassa virus $[2,16]$.

\section{Mode of Transmission}

Once the multimammate rat becomes infected with the Lassa virus, it can shed the virus in its urine and excreta for the rest of its life [17]. There are numerous complex interactions that occur between humans and the environment that make the Lassa virus more likely to crossover from the animal reservoir to the human host [2]. For instance, in tropical West Africa, there are seasonal patterns accompanying the risk of getting infected with the Lassa virus, as hospitalized cases of Lassa fever are at the highest during the dry season [18]. Since reduced rainfall is correlated with a reduction of the probability of flooding, multimammate rats have a habitat that is more conducive to breeding in the dry season. Zoonotic spillovers are thought to occur as a result of humans interacting with rodent urine and/or excreta through contaminated food or inhaling aerosols from desiccated urine or feces [2]. Person-to-person transmission of the virus is also possible, particularly in rural health facilities, where health workers come in contact with the blood, pharyngeal secretions, or urine of infected individuals [17]. In addition, there have also been cases of Lassa virus infection occurring through sexual transmission as the virus can remain in the semen for up to 3 months after infection [19]. There is no evidence of airborne transmission of the Lassa virus from infected to healthy individuals [3]. The people who have the highest risk of getting infected with the Lassa virus are people resident in rural areas where the multimammate rat is in abundance. Health workers also have a high risk of getting infected when treating infected individuals, particularly when they do not practice, infection prevention and control measures to limit the nosocomial spread of the Lassa virus [3].

\section{Symptoms of Lassa Fever}

Lassa fever presents numerous symptoms that make it difficult for local physicians to make an accurate diagnosis, particularly those who are not familiar with the disease $[3,10]$. The Lassa virus incubates within the infected persons from 6 to 21 days before there is a gradual manifestation of mild symptoms such as low-grade fever, headache, malaise, as well as general weakness. About $80 \%$ of all Lassa virus infections are subclinical and do not progress beyond mild malaise [10]. Between 4 and 7 days after the onset of the mild symptoms, up to $20 \%$ of infected individuals experience more severe symptoms such as hemorrhage, persistent vomiting, hypotension, edema around the neck and face, respiratory distress, as well as hypovolemic shock [10]. Over $25 \%$ of Lassa fever cases result in some degree of deafness, with normal hearing gradually returning 30-90 days after recovering from the viral infection. Convalescing individuals also experience some form of hair loss and impaired gait. In more severe cases, there is also the presence of fluid within the lungs, bleeding from the mouth, nose, vagina, and alimentary canal. There are also some cases of Lassa fever where infected individuals have abnormal quantities of protein in their urine [3]. Death typically occurs from organ failure within 10-14 days from the manifestation of more severe symptoms, which also include seizures, tremors, disorientation, as well as coma [3]. During pregnancy, especially the third trimester, over $80 \%$ of all Lassa fever cases are fatal [10].

\section{Diagnosis and Treatment Options}

Lassa fever may be diagnosed by utilizing enzyme-linked immunosorbent serologic assays which useful in detecting IgM and IgG antibodies, as well as the Lassa antigen. However, the most common and effective way of detecting the virus is through reverse transcription-polymerase chain reaction (RT-PCR). Postmortem diagnosis can also be done through immunohistochemistry carried out on tissue specimens that 
have been fixed with formalin [6]. Antigen detection tests, as well as virus isolation by cell culture, have also been used in diagnosing Lassa fever in the laboratory [3]. Ribavirin is currently used in treating individuals with cases of Lassa fever, as well as for the purpose of post-exposure prophylaxis $[9,20]$.

\section{Current Lassa Fever Trends}

The past decade has seen increases in the number of suspected and confirmed cases of Lassa fever infections [21]. These increases can be ascribed to the shift from sourcing information about Lassa fever from literature and research publications, to obtaining knowledge from actual Lassa fever case reports from disease surveillance reports [2]. In recent times, there has been an increase in the incidence of Lassa fever cases in regions where the Lassa virus is endemic. This may be attributed to the increasing surveillance for Lassa fever in West Africa [2]. A good example of this is the upward trend of Lassa fever cases in Nigeria from 430 suspected cases in 2015 to 900,700 , and 1081 suspected cases of the disease in 2016, 2017, and 2018 , respectively $[3,22]$.

Through ecological niche modeling, it has been reported that there is a correlation between certain environmental variables (such as rainfall, human population density, and rice yields) with outbreaks of the Lassa virus infection [5]. West African climate projections predict that there would be an increase in both temperature and rainfall, which is expected to lead to an increase in the likelihood of the multimammate rat thriving in the West African subregion, consequently increasing the chances of human infection with the Lassa virus [2]. Furthermore, land use projections in West Africa have yielded different predictions concerning the significance of land use on the future incidence of Lassa fever cases. On the one hand, there are future expectations that land use for both subsistence and large-scale farming will be commonplace. This may make it much easier for the Lassa virus to spread, as there farming activities will bring humans in closer contact with infected aerosols from infected multimammate rat [2]. However, on the other hand, there are projections that have proposed that an increase in commercial agriculture in future West Africa could potentially reduce risks of infection with Lassa virus as mechanized farming will reduce the number of individuals who may have encountered the infected vector in its natural habitat [23], consequently leading to a decrease in Lassa virus infections.

Researchers are also trying to understand the role that global warming and general climate change play in the increased number Lassa fever cases because of increased transmissibility of the virus [24]. A possible explanation is that warmer temperature could allow the growth of more crops, which, in turn, would provide food to sustain more multimammate rats [25]. Multimammate rats can grow exponentially at rates that are 10-20 times that of humans, and as a result, favorable environmental conditions would make humans much more likely to be infected with the Lassa virus [25].

In addition, over time, certain groups of people from endemic regions may be able to develop some form of resistance to Lassa virus. This hypothesis has been proven to some extent by data obtained from the Yoruba people in Nigeria who seemed to have developed some degree of genetic resistance to the Lassa virus in comparison to other endemic regions [26].

\section{Possible Reduction Solutions}

Community hygiene is one of the most potent ways to curtail the rampage of Lassa fever in endemic areas [3]. According to the CDC [6], humans can prevent Lassa virus infection by avoiding all forms of contact with the multimammate rats, particularly in the regions where Lassa virus is endemic. It is also recommended that food should be stored in rodent-proof containers, while also ensuring that the environments are kept clean to prevent the rats from breeding in houses. It is expected that this would reduce the likelihood of humans being infected with the Lassa virus. Furthermore, people should be discouraged from eating rodents, as the process of trapping, catching, or cleaning an infected rodent could lead to a human being infected with the Lassa virus. While setting traps to catch multimammate rats might play a role in reducing the number of animals available to act as reservoirs for the Lassa virus, it is not practical to do so because the multimammate rats are dispersed across West Africa [6].

Furthermore, there are instances where care needs to be provided for a person infected with the Lassa virus. According to the CDC [6], it is expedient to take preventive measures to limit the spread of the infection. Some of the measures that can be taken include setting up biological containment conditions, wearing protective clothes (such as masks, gloves, gowns, and goggles), proper sterilization of equipment, as well as the isolation of infected individuals to prevent the spread of the virus to unprotected persons.

There are also instances where individuals who travel to regions where Lassa fever is endemic export the virus to their home countries and/or other countries. Individuals returning to their home countries with fever symptoms, especially if they have been traveling from West Africa, should be tested for Lassa fever as soon as possible [3].

Finally, people who are resident in endemic regions, particularly those in the rural areas, should be educated on effective means of reducing the rodent population, so as to limit and preempt the spread of Lassa fever [6].

\section{Conclusion}

As the world becomes increasingly connected, viral diseases, such as Lassa fever, once endemic to a region can be easily transmitted to other parts of 
the world, thus increasing the likelihood of a global pandemic. To reduce the probabilities of such events playing out, deliberate and concerted efforts must be applied towards both understanding Lassa fever and limiting infection rates. This paper provided an overview of the causes and effects of Lassa virus infection, as well as proven reduction strategies useful for managing such infections in the event that they occur.

\section{Authors' Contributions}

$\mathrm{PT}$ and $\mathrm{NH}$ conceived the original idea. PT took the lead in writing the manuscript. Both authors read and approved the final manuscript.

\section{Acknowledgments}

This research did not receive any financial support from funding agencies in the public, commercial, or not-for-profit sectors. The authors would like to thank Dr. Jamie Johnson for sparking our initial interest in global epidemiology.

\section{Competing Interests} interests.

The authors declare that they have no competing

\section{Publisher's Note}

Veterinary World (Publisher of International Journal of One Health) remains neutral with regard to jurisdictional claims in published institutional affiliation.

\section{References}

1. Lehmann, C., Kochanek, M., Abdulla, D., Becker, S., Böll, B., Bunte, A. and Feldt, T. (2017) Control measures following a case of imported Lassa fever from Togo, North Rhine Westphalia, Germany, 2016. Euro Surveill., 22(39): 17-88.

2. Gibb, R., Moses, L.M., Redding, D.W. and Jones, K.E. (2017) Understanding the cryptic nature of Lassa fever in West Africa. Pathog. Glob. Health, 111(6): 276-288.

3. World Health Organization. (2017) Lassa Fever. World Health Organization, Geneva. Available from: http://www. who.int/mediacentre/factsheets/fs 179/en. Retrieved on 04-06-2020.

4. Mylne, A.Q., Pigott, D.M., Longbottom, J., Shearer, F., Duda, K.A., Messina, J.P. and Hay, S.I. (2015) Mapping the zoonotic niche of Lassa fever in Africa. Trans. R. Soc. Trop. Med. Hyg., 109(8): 483-492.

5. Fichet-Calvet, E. and Rogers, D.J. (2009) Risk maps of Lassa fever in West Africa. PLoS Negl. Trop. Dis., 3(3): e388.

6. Centers for Disease Control and Prevention. (2015) Lassa Fever. Centers for Disease Control and Prevention, Atlanta, Georgia. Available from: https://www.cdc.gov/vhf/lassa/ index.html. Retrieved on 04-06-2020.

7. Peterson, A.T., Moses, L.M. and Bausch, D.G. (2014) Mapping transmission risk of Lassa fever in West Africa: The importance of quality control, sampling bias, and error weighting. PLoS One, 9(8): e100711.

8. Ogbu, O., Ajuluchukwu, E. and Uneke, C.J. (2007) Lassa fever in West African sub-region: An overview. J. Vector Borne Dis., 44(1): 1-11.

9. Olayiwola, J.O. and Bakarey, A.S. (2017) Epidemiological trends of Lassa Fever Outbreaks and Insights for Future Control in Nigeria. Int. J. Trop. Dis. Health, 24(4): 1-14.

10. Sogoba, N., Feldmann, H. and Safronetz, D. (2012) Lassa fever in West Africa: Evidence for an expanded region of endemicity. Zoonoses Public Health, 59(s2): 43-47.

11. Asogun, D.A., Adomeh, D.I., Ehimuan, J., Odia, I., Hass, M., Gabriel, M. and Ehiane, P.E. (2012) Molecular diagnostics for Lassa fever at Irrua specialist teaching hospital, Nigeria: Lessons learnt from two years of laboratory operation. PLoS Negl. Trop. Dis., 6(9): e1839.

12. Bowen, M.D., Peters, C.J. and Nichol, S.T. (1997) Phylogenetic analysis of the arenaviridae: Patterns of virus evolution and evidence for cospeciation between arenaviruses and their rodent hosts. Mol. Phylogenet. Evol., 8(3): 301-316.

13. Lozano, M.E., Posik, D.M., Albarino, C.G., Schujman, G., Ghiringhelli, P.D., Calderon, G. and Romanowski, V. (1997) Characterization of arenaviruses using a family-specific primer set for RT-PCR amplification and RFLP analysis: Its potential use for detection of uncharacterized arenaviruses. Virus Res., 49(1): 79-89.

14. Granjon, L., Cosson, J.F., Quesseveur, E. and Sicard, B. (2005) Population dynamics of the multimammate rat Mastomys huberti in an annually flooded agricultural region of central Mali. J. Mammal., 86(5): 997-1008.

15. Olayemi, A., Cadar, D., Magassouba, N.F., Obadare, A., Kourouma, F., Oyeyiola, A. and Jérôme, H. (2016) New hosts of the Lassa virus. Sci. Rep., 6(1): 1-6.

16. Gryseels, S., Goüy de Bellocq, J., Makundi, R., Vanmechelen, K., Broeckhove, J., Mazoch, V. and Baird, S.J.E. (2016) Genetic distinction between contiguous urban and rural multimammate mice in Tanzania despite gene flow. J. Evol. Biol., 29(10): 1952-1967.

17. Saka, M.J., Gubio, A.B., Yennan, S.K., Saka, A.O. and Oyemakinde, A. (2017) Lassa fever epidemic in Nigeriaoutbreak investigation, risk factors and empirical analysis from 2012 To 2016. J. Community Public Health Nurs., 3(2): 170.

18. Iacono, G.L., Cunningham, A.A., Fichet-Calvet, E., Garry, R.F., Grant, D.S., Leach, M. and Webb, C.T. (2016) A unified framework for the infection dynamics of zoonotic spillover and spread. PLoS Negl. Trop. Dis., 10(9): e0004957.

19. GOV.UK. (2016) Lassa Fever: Origins, Reservoirs, Transmission and Guidelines. Available from: https://www. gov.uk/guidance/lassa-fever-origins-reservoirs-transmission-and-guidelines. Retrieved on 08-05-2018.

20. Bausch, D.G., Hadi, C.M., Khan, S.H. and Lertora, J.J. (2010) Review of the literature and proposed guidelines for the use of oral ribavirin as postexposure prophylaxis for Lassa fever. Clin. Infect. Dis., 51(12): 1435-1441.

21. Shaffer, J.G., Grant, D.S., Schieffelin, J.S., Boisen, M.L., Goba, A., Hartnett, J.N. and Momoh, M. (2014) Lassa fever in post-conflict Sierra Leone. PLoS Negl. Trop. Dis., 8(3): e2748.

22. National Centre for Disease Control. (2019) An Update of Lassa Fever Outbreak in Nigeria. Available from: https:// ncdc. gov.ng $/$ diseases $/$ sitreps $/$ ? cat $=5 \&$ name $=\mathrm{An} \% 20$ update $\% 20$ of $\% 20$ Lassa $\% 20$ fever $\% 20$ outbreak $\% 20 \mathrm{in} \% 20$ Nigeria. Retrieved on 21-12-2019.

23. Massawe, A.W., Rwamugira, W., Leirs, H., Makundi, R.H. and Mulungu, L.S. (2007) Do farming practices influence population dynamics of rodents? A case study of the multimammate field rats, Mastomys natalensis, in Tanzania. Afr. J. Ecol., 45(3): 293-301.

24. Clegg, J.C. (2009) Influence of climate change on the incidence and impact of Arenavirus diseases: A speculative assessment. Clin. Microbiol. Infect., 15(6): 504-509.

25. Belluz, J. (2018) Why Lassa, an Ebola-like Fever, Has Exploded in Nigeria. Available from: https://www.vox. com/science-and-health/2018/3/9/17092624/lassa-fever-virus-outbreak-symptoms-nigeria. Retrieved on 12-05-2018.

26. Andersen, K.G., Shylakhter, I., Tabrizi, S., Grossman, S.R., Happi, C.T. and Sabeti, P.C. (2012) Genome-wide scans provide evidence for positive selection of genes implicated in Lassa fever. Philos. Trans. R. Soc. B Biol. Sci., 367(1590): 868-877. 